\title{
Investigation of oscillations of platform on isotropic supports excited by a pendulum
}

\author{
Gennadiy Filimonikhin ${ }^{1, *}$, Volodymyr Yatsun $^{1}$, and Irina Filimonikhina ${ }^{1}$ \\ ${ }^{1}$ Central Ukrainian National Technical University, 25006, Kropyvnytskyi, Universytetskyi Ave., 8, \\ Ukraine
}

\begin{abstract}
Within the framework of a flat model, steady-state modes of motion of a system composed of a platform on isotropic elastic-viscous supports, a shaft on a platform, and a pendulum freely mounted on a shaft are investigated. The developed methodology was used in the studies, based on the energy method, the theory of bifurcations of motions, and the idea of a parametric solution to the problem. All steady-state modes of motion were found. It is established that these are modes of the pendulum jamming. Each mode is characterized by a corresponding jamming frequency. Depending on the velocity of rotation of the shaft, there may be one or three possible jamming frequencies. When there is only one jamming frequency, the corresponding mode of motion is globally asymptotically stable. When there are three jamming frequencies, locally asymptotically stable modes with the smallest and highest jamming frequencies of the pendulum. The smallest jamming frequency of the pendulum is close to resonance. This mode can be used to excite resonant vibrations in vibrating machines. The highest jamming frequency of a pendulum is close to the shaft rotation velocity. This mode can be used to excite non-resonant vibrations in vibrating machines.
\end{abstract}

\section{Introduction}

In the mining and oil industries, vibration screens, sieves, mills, conveyors, etc. are widely used. Among these vibration machines are the most energy-efficient resonant vibration machines. To excite purely resonant vibrations, a method based on the use of pendulum, ball or roller auto-balancers as a vibration exciter can be used [1].

It should be noted that, depending on the parameters of the system, a vibration (rotor) machine with an auto-balancer can carry out motion modes corresponding to:

- auto-balancing or synchronous rotation of weights with the rotor [2];

- jamming of pendulums [3], balls (rollers) or pendulums [4, 5], balls [6, 7] at the resonant rotor speed (caused by the Sommerfeld effect)

- parametric and other vibrations of weights relative to the rotor [8].

Therefore, the problem arises of the proper selection of the parameters of the vibration machine for excitation and ensuring the stability of the resonant modes of motion. The analytical solution of such problems is associated with significant mathematical difficulties

"Corresponding author: filimonikhin@ukr.net 
caused by both a large number of different modes of motion of the rotor machine [2, 4] and significant nonlinearity of the problems [2-8]. Therefore, it is important to develop a methodology for the numerical study of steady-state modes of motion of vibrating machines with auto-balancer. Below, such a technique is developed using the example of a vibration machine, in which the pendulum is freely mounted on a rotating shaft with respect to an elastic-viscous fixed platform.

The aim of research is to develop, using the specified vibration machine, as an example, a methodology for studying possible steady-state motion modes and assessing their stability. This will allow to solve similar problems for other vibration machines, in particular - single-mass and multi-mass, with different kinematics of the motion of the vibrating platform.

To achieve the aim, the following tasks were set:

- develop a methodology for the study of the steady-state motions of the specified vibration machine;

- using the methodology, find all the steady-state modes of motion of the vibration machine with specific values of its parameters, asses the stability of various modes;

- determine the applicability of various jamming modes of the pendulum to excite platform vibrations.

\section{Methods of searching for all possible steady-state modes of system motion and studying their stability}

To build a mechanical-mathematical model of a mechanical system, elements of the theory of machines with passive auto-balancer [1-8], classical mechanics [9] are used.

The equation of the steady-state motions of the system is obtained from the application of the energy approach described in [2] on the generalized potential and the linear part of the dissipative function.

The search for all possible modes of pendulum jamming is carried out using the results obtained in [5] using a special technique. The occurrence and disappearance of various modes of jamming is interpreted from the point of view of the theory of bifurcations of motions [10]. According to this theory, the pendulum jamming modes can acquire or lose stability only at the points of bifurcations of motions. From an energetic point of view, when several jamming modes are nucleated, there is a stable mode, in which the potential is brought to the least value [2]. Solutions of nonlinear equations are sought in a parametric form.

A research technique has been developed that is verified by specific numerical calculations.

\section{Results and discussion}

\subsection{Mechanical-mathematical model of the system}

\subsubsection{Description of the mechanical-mathematical model of the system}

The dynamics of the system is studied in the framework of a flat model (Fig. 1).

A platform of mass $m_{p l}(\mathrm{~kg})$ is contained by isotropic elastic-viscous supports (Fig. 1, a). Stiffness and damping coefficients in the supports $-\widetilde{c}(\mathrm{~N} / \mathrm{m}), \tilde{b}(\mathrm{~N} \cdot \mathrm{s} / \mathrm{m})$. The platform makes translational motion. An absolutely rigid shaft of mass $m_{s}(\mathrm{~kg})$ is mounted on the platform. The shaft axis is perpendicular to the platform and passes through its center of mass. The shaft rotates relative to the platform with a constant angular velocity $\omega(\mathrm{rad} / \mathrm{s})$. A pendulum 
is mounted on the shaft (Fig. 1, b), which mass is $m_{p n}(\mathrm{~kg})$, physical length $l(\mathrm{~m})$ and the main central axial moment of inertia $I_{C}^{(p n)}\left(\mathrm{kg} \cdot \mathrm{m}^{2}\right)$. Let's neglect the forces of weight.

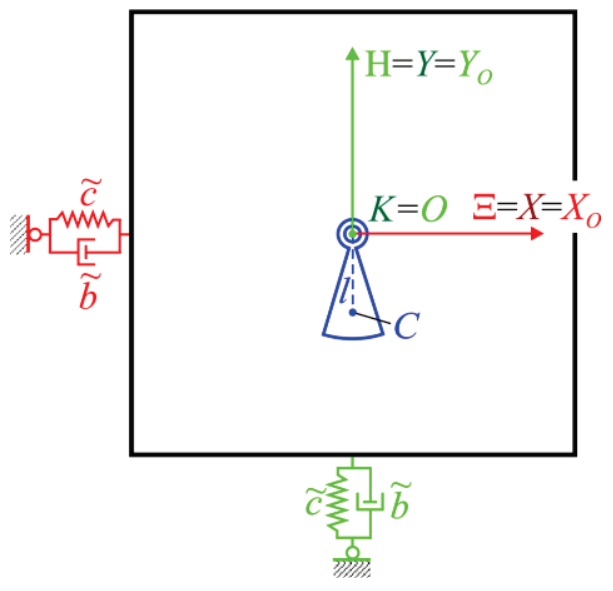

$\mathrm{a}$

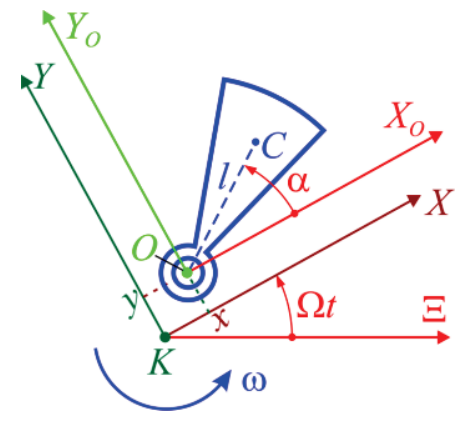

b

Fig. 1. A flat model of the system: $a-a$ platform on isotropic elastic-viscous supports; $b$ - kinematics of the pendulum motion.

With a stationary system, the center of the shaft, point $O$ is at point $K$. During the motion of the platform, the shaft, point $O$, deviates from point $K$, and a restoring force and the force of viscous resistance begin to act on the platform.

To describe the motion of the system, let's use the following axis systems:

$-K \Xi H$ - right system of fixed rectangular axes;

$-K X Y$ - right system of moving rectangular axes, which rotates around the axis of rotation (point $K$ ) with a constant angular velocity $\Omega(\mathrm{rad} / \mathrm{s})$ synchronously with the pendulum;

$-O X_{O} Y_{O}$ - right system of movable rectangular axes, which starts in the center of the shaft and parallel to the $K X Y$ axis system.

The rotation angle of the $K X Y$ axis system around the point $K$ is $\Omega t$, where $t$ (s) is the time. Shaft angle $\omega t$. The position of the pendulum is determined with respect to the $O X_{O} Y_{O}$ axis system by a constant angle $\alpha(\mathrm{rad})$. When the pendulum rotates relative to the shaft, the moment of viscous resistance forces $\widetilde{\beta} l^{2}(\omega-\Omega)$ acts on it, where $\widetilde{\beta}(\mathrm{N} \cdot \mathrm{s} / \mathrm{m})$ - coefficient of viscous resistance forces and $(\omega-\Omega)$ - angular velocity of rotation of the pendulum relative to the shaft.

For the investigated system

$$
M_{\Sigma}=m_{p l}+m_{s}+m_{p n}, \quad \omega_{0}=\sqrt{c / M_{\Sigma}}
$$

where $M_{\Sigma}(\mathrm{kg})$ - total system mass, $\omega_{0}(\mathrm{rad} / \mathrm{s})$ - resonant rotation velocity of the shaft.

\subsubsection{Generalized potential, dissipative function, and equations of steady-state system motions}

System potential energy

$$
V=\widetilde{c}\left(x^{2}+y^{2}\right) / 2,(\mathrm{~N} \cdot \mathrm{m})
$$


On steady-state motion, the kinetic energy of the shaft and platform is the sum of the kinetic energies of the translational motion of the shaft and platform together with the center of mass (point $O$ ) and the rotation of the shaft around the center of mass [9]:

$$
T_{p l, s}=\left(m_{p l}+m_{s}\right)\left(x^{2}+y^{2}\right) \Omega^{2} / 2+I_{O}^{(s)} \omega^{2} / 2,(\mathrm{~N} \cdot \mathrm{m})
$$

where $I_{O}^{(s)}\left(\mathrm{kg} \cdot \mathrm{m}^{2}\right)$ - axial moment of inertia of the shaft relative to the longitudinal axis.

On steady-state motion, the pendulum stops moving relative to the moving axes. Its kinetic energy can be represented as

$$
T_{b}=I_{K}^{(p n)} \Omega^{2} / 2,(\mathrm{~N} \cdot \mathrm{m}),
$$

where $I_{K}^{(p n)}\left(\mathrm{kg} \cdot \mathrm{m}^{2}\right)$ - axial moment of inertia of the pendulum relative to point $K$.

In turn

$$
\begin{aligned}
I_{K}^{(p n)} & =I_{C}^{(p n)}+m_{p n}\left[(x+l \cos \alpha)^{2}+(y+l \sin \alpha)^{2}\right]= \\
& =I_{O}^{(p n)}+m_{p n}\left[\left(x^{2}+y^{2}\right)+2 x l \cos \alpha+2 y l \sin \alpha\right]
\end{aligned}
$$

where $I_{O}^{(p n)}=I_{C}^{(p n)}+m_{p n} l^{2}\left(\mathrm{~kg} \cdot \mathrm{m}^{2}\right)$ - axial moment of inertia of the pendulum relative to point $O$.

Thus, the kinetic energy of the system in steady-state motion

$$
\begin{gathered}
T_{0}=T_{p l, s}+T_{p n}= \\
=M_{\Sigma}\left(x^{2}+y^{2}\right) \Omega^{2} / 2+I_{O}^{(s)} \omega^{2} / 2+\left[I_{O}^{(p n)}+2 m_{p l}(x l \cos \alpha+y l \sin \alpha) \Omega^{2} / 2 .\right.
\end{gathered}
$$

The generalized potential (let's omit the constant $I_{O}^{(s)} \omega^{2} / 2$ ):

$$
\Pi=V-T_{0}=\left(c-\Omega^{2} M_{\Sigma}\right)\left(x^{2}+y^{2}\right) / 2-\left[I_{O}^{(p n)}+2 m_{p l}(x l \cos \alpha+y l \sin \alpha)\right] \Omega^{2} / 2,(\mathrm{~N} \cdot \mathrm{m}) .
$$

Dissipative function

$$
D=b\left[(\dot{x}-\Omega y)^{2}+(\dot{y}+\Omega x)^{2}\right] / 2+\beta l^{2}(\omega-\Omega-\dot{\alpha})^{2} / 2,(\mathrm{~N} \cdot \mathrm{m} / \mathrm{s}) .
$$

Component linear with respect to generalized velocities

$$
D_{1}=b \Omega(-\dot{x} y+\dot{y} x)-\beta l^{2}(\omega-\Omega) \dot{\alpha},(\mathrm{N} \cdot \mathrm{m} / \mathrm{s}) .
$$

The equations of stationary motions of a mechanical system have the form [2]

$$
\begin{gathered}
\widetilde{L}=\partial \Pi / \partial \alpha+\partial D_{1} / \partial \dot{\alpha}=\widetilde{\beta} l^{2}(\Omega-\omega)+m_{p l} l \Omega^{2}(\tilde{x} \sin \tilde{\alpha}-\tilde{y} \cos \tilde{\alpha})=0, \\
\widetilde{L}_{x}=\partial \Pi / \partial x+\partial D_{1} / \partial \dot{x}=\left(\tilde{c}-M_{\Sigma} \Omega^{2}\right) \tilde{x}-\tilde{b} \Omega \tilde{y}-\Omega^{2} m_{p l} l \cos \widetilde{\alpha}=0 \\
\widetilde{L}_{y}=\partial \Pi / \partial y+\partial D_{1} / \partial \dot{y}=\left(\tilde{c}-M_{\Sigma} \Omega^{2}\right) \tilde{y}-\tilde{b} \Omega \tilde{x}-\Omega^{2} m_{p l} l \sin \tilde{\alpha}=0 .
\end{gathered}
$$




\subsubsection{The statement of the problem of studying the steady-state modes of motion of the system in a dimensionless form}

Let's introduce dimensionless time, variables, and parameters

$$
\tau=\Omega t, u=\frac{x}{l}, v=\frac{y}{l}, n=\frac{\omega}{\omega_{0}}, \beta=\frac{\widetilde{\beta}}{m_{p l} \omega_{0}}, v=\frac{\Omega}{\omega_{0}}, b=\frac{\widetilde{b}}{\omega_{0} M_{\Sigma}}, \varepsilon=\frac{m_{p l}}{M_{\Sigma}}
$$

From (10) let's obtain the following dimensionless equations of stationary motions

$$
\begin{gathered}
\widetilde{L}^{(0)}=\beta(v-n)+v^{2}(\tilde{u} \sin \tilde{\alpha}-\tilde{v} \cos \tilde{\alpha})=0, \\
L_{u}^{(0)}=\tilde{u}\left(1-v^{2}\right)-b v \widetilde{v}-\varepsilon v^{2} \cos \widetilde{\alpha}=0, \quad L_{v}^{(0)}=\left(1-v^{2}\right) \widetilde{v}+b v \tilde{u}-\varepsilon v^{2} \sin \widetilde{\alpha}=0 .
\end{gathered}
$$

Dimensional generalized potential in steady-state motion

$$
\Pi^{*}=\Pi /\left(c l^{2}\right)=\left(1-v^{2}\right)\left(\tilde{u}^{2}+\widetilde{v}^{2}\right) / 2-\left[\tilde{I}_{O}^{(p n)}+2 \varepsilon(\tilde{u} \cos \alpha+\widetilde{v} \sin \alpha)\right] v^{2} / 2,
$$

where

$$
\widetilde{I}_{O}^{(p n)}=I_{O}^{(p n)} /\left(M_{\Sigma} l^{2}\right)=m_{p n} l^{2} /\left(M_{\Sigma} l^{2}\right)=m_{p n} / M_{\Sigma}
$$

From the last two equations in (12) let's find

$$
\varepsilon v^{2} \cos \tilde{\alpha}=\tilde{u}\left(1-v^{2}\right)-b v \widetilde{v}, \varepsilon v^{2} \sin \tilde{\alpha}=\left(1-v^{2}\right) \widetilde{v}+b v \tilde{u} .
$$

Substituting this into (7), let's obtain the dimensionless reduced generalized potential

$$
\widetilde{\Pi}^{*}=-\left(1-v^{2}\right)\left(\widetilde{u}^{2}+\widetilde{v}^{2}\right) / 2-\widetilde{I}_{O}^{(p n)} v^{2} / 2
$$

It is more convenient to use the potential $\widetilde{\Pi}^{*}$ for assessing the stability of various steady-state modes of motion, because it does not depend on an undefined parameter $\widetilde{\alpha}$ unlike $\Pi^{*}$

Let's introduce the angle $\widetilde{\vartheta}$ between the vector $\overrightarrow{K O}$ (the shaft displacement vector) and the $X$ axis. Then

$$
\cos \tilde{\vartheta}=\tilde{u} / \tilde{p}, \sin \tilde{\vartheta}=\widetilde{v} / \tilde{p}, \tilde{\rho}=\sqrt{\tilde{u}^{2}+\widetilde{v}^{2}}
$$

and the equations of steady-state motions (12) are transformed into:

$$
\begin{gathered}
\widetilde{L}^{(0)}=\beta(v-n)-v^{2} \widetilde{\rho} \sin \widetilde{\varphi}=0, \\
\widetilde{u} \widetilde{L}_{u}^{(0)}+\widetilde{v} \widetilde{L}_{v}^{(0)}=\left(1-v^{2}\right) \widetilde{\rho}^{2}-\varepsilon \widetilde{\rho} v^{2} \cos \widetilde{\varphi}=0, \widetilde{u} L_{u}^{(0)}-\widetilde{v} \widetilde{L}_{v}^{(0)}=\widetilde{b} v \widetilde{\rho}^{2}+\varepsilon \widetilde{\rho} v^{2} \sin \widetilde{\varphi}=0,
\end{gathered}
$$

where

$$
\widetilde{\varphi}=\widetilde{\vartheta}-\widetilde{\alpha}
$$

The resulting system of three nonlinear equations with respect to three unknowns $\tilde{\rho}, v$, 
$\tilde{\varphi}$. System (18), up to the notation, coincided with the equations obtained in [5] for a balanced rotor with isotropic bearings, on which shaft a pendulum is mounted. Therefore, in the future let's use the results of this work.

\subsection{Study of steady-state motions of the system}

\subsubsection{Formal solution to the problem}

According to the results of [5], there is no mode of motions on which the pendulum rotates synchronously with the shaft in the considered vibration machine. The machine has only modes in which the pendulum is behind the shaft.

The system of equations (18) is solved as follows [5]. From polynomial

$$
P(v)=\chi v^{5}-(n-v)\left[\left(1-v^{2}\right)^{2}+b^{2} v^{2}\right]=a_{0} v^{5}+a_{1} v^{4}+a_{2} v^{3}+a_{3} v^{2}+a_{4} v+a_{5}=0,
$$

where

$$
\chi=\varepsilon b / \beta, a_{0}=1+\chi, a_{1}=-n, a_{2}=-\left(2-b^{2}\right), a_{3}=n\left(2-b^{2}\right), a_{4}=1, a_{5}=-n .
$$

the frequencies $v_{i}$, at which the pendulum is jamming are determined. The polynomial is easier to solve in parametric form:

$$
n(v)=v+\chi v^{5} /\left[\left(1-v^{2}\right)^{2}+b^{2} v^{2}\right], v \in(0,+\infty) .
$$

Then, for a particular pendulum jamming frequency,

$$
\begin{gathered}
\tilde{\rho}=\sqrt{\varepsilon \beta(n-v) /(v b)}=\chi \beta v^{2} /\left[b \sqrt{\left(1-v^{2}\right)^{2}+b^{2} v^{2}}\right], \\
\widetilde{\varphi}=\left\{\begin{array}{l}
\gamma, \gamma \leq 0 ; \\
\gamma-\pi, \gamma>0,
\end{array} \quad \gamma=\arctan \left(\frac{b v}{v^{2}-1}\right), \tilde{\alpha}=\widetilde{\vartheta}-\tilde{\varphi}, \quad \tilde{u}=\tilde{\rho} \cos \widetilde{\vartheta}, \tilde{v}=\tilde{\rho} \sin \widetilde{\vartheta}\right.
\end{gathered}
$$

Taking into account (17), (23), the generalized potential (16) is calculated by the formula

$$
\widetilde{\Pi}^{*}=-\left(1-v^{2}\right) \beta^{2} \chi^{2} v^{4} /\left\{2 b^{2}\left[\left(1-v^{2}\right)^{2}+b^{2} v^{2}\right]\right\}-\widetilde{I}_{O}^{(p n)} v^{2} / 2
$$

Let's note that the values $\widetilde{\alpha}, \widetilde{u}, \widetilde{v}$ are calculated for a specific (but any) value of the parameter $\vartheta$.

\subsubsection{Numerical studies of system performance}

Let's consider the dimensionless angular velocity of rotation of the shaft $n$ as a bifurcation parameter. As $n$ changes from 0 to $+\infty$, the roots of equation (20) will change. Let's look for the real roots and bifurcation angular velocities of rotation of the shaft (in which various modes of jamming arise or disappear).

Since it is difficult to find the roots of the fifth polynomial, let's solve the problem parametrically. According to the parameter, let's take the frequency of the pendulum jamming. Then the solution of equation (20) in parametric form has the form (22).

In Figure 2, a graph of the function $n(v)$ is constructed in the $(n, v)$ plane for a different 
ratio of smallness between the parameters $\chi$ and $b[5]$.

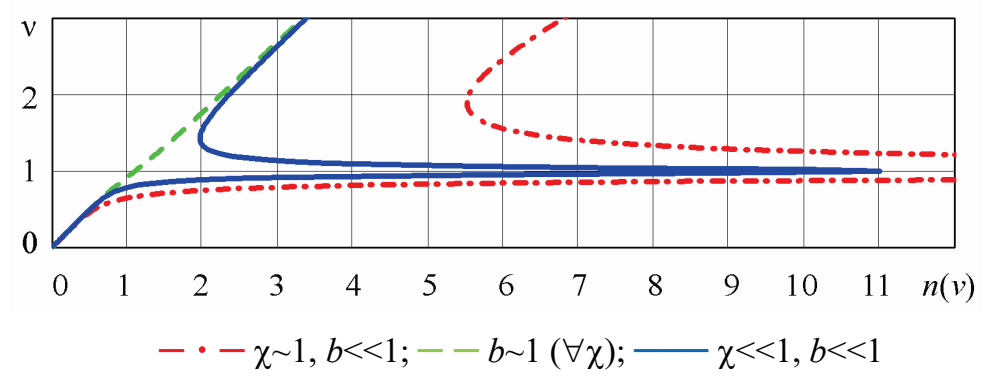

Fig. 2. The dependence of the number and conditions for the existence of frequencies of a jammed pendulum on the ratio of smallness between the parameters $\chi$ and $b$.

Figure 2 shows that when $\chi \sim 1, b<<1$ or $\chi<<1, b<<1$ in the system, depending on the rotation velocity of the shaft, there are one or three possible frequencies of jamming of the pendulum. At $b \sim 1$ in the system, there is the only possible frequency for the jamming of the pendulum close to the shaft rotation velocity.

In the future, let's consider the case of small viscous drag forces in the supports [5]. Let's introduce for this case two bifurcation velocities of rotation of the shaft. The number of possible frequencies of the jamming of the pendulum changes in the case of their transition. At the same time $1<n_{1}<<n_{2}$ and at shaft velocities:

- less than $n_{1}\left(0<n<n_{1}\right)$ there is a unique jamming frequency $v_{1}$, with $0<v_{1}<1$;

- exceeding $n_{1}$, but less then $n_{2} \quad\left(n_{1}<n<n_{2}\right)$ there are three jamming frequencies $v_{1,2,3}$, such that $0<v_{1}<v_{2}<v_{3}<n$ (and always $1<v_{2}<v_{3}$ );

- exceeding $n_{2}\left(n>n_{2}\right)$, there is a single jamming frequency $v_{3}$, such that $1<<v_{3}<n$.

At point $n_{1}$, the jamming modes $v_{2}$ and $v_{3}$ appear, and at point $n_{2}$, the jamming modes $v_{1}$ and $v_{2}$ disappear (through merging). According to the theory of bifurcations of motions, various modes of jamming can acquire or lose stability only when passing through the points of bifurcations of motions [10]. When several motions arise, only the one on which the reduced potential is the least can be stable [2].

Figure 2 shows that in a critical case (nucleation or fusion of various jamming modes)

$$
d n(v) / d v=F(v) /\left[\left(1-v^{2}\right)^{2}+b^{2} v^{2}\right]^{2}=0,
$$

where

$$
F(v)=(1+\chi) v^{8}-\left(2-b^{2}\right)(2+3 \chi) v^{6}+\left[\left(2-b^{2}\right)^{2}+2+3 \chi\right] v^{4}-2\left(2-b^{2}\right) v^{2}+1 .
$$

The carried out studies allow to conclude that the excitation of resonant oscillations is possible only in the case of small external and internal resistance forces.

\subsubsection{Calculation algorithm for studying the quantity, conditions of existence and stability of various modes of pendulum jamming}

The results obtained with a qualitative assessment of the behavior of the system allow to develop such a computational algorithm.

1. The critical frequencies of jamming of the load are determined from the equation $F(v)=0$. From the graphs in Figure 2 shows that there are two such frequencies: 
$v_{c 1}, v_{c 2}: v_{c 2}>v_{c 1}$.

2. Using the formula (22), two bifurcation angular rotational velocities of the rotor are determined $n_{i}=n\left(v_{c i}\right), / i=1,2 /: n_{1}<n_{2}$.

3. For each jamming mode, according to formula (22), the corresponding rotor velocities are calculated in parametric form

$$
n_{1}(v)=n(v), v \in\left[0, v_{c 1}\right] ; n_{2}(v)=n(v), v \in\left[v_{c 1}, v_{c 2}\right] ; n_{3}(v)=n(v), v \in\left[v_{c 2},+\infty\right)
$$

Based on the results of calculations in the $(n, v)$ plane, graphs $\left(n_{1}(v), v\right), / i=1,2,3 /$ are constructed.

4. For each jamming mode, according to formula (26), the corresponding vibration amplitudes are calculated in a parametric form

$$
\rho_{1}(v)=\rho(v), v \in\left[0, v_{c 1}\right] ; \rho_{2}(v)=\rho(v), v \in\left[v_{c 1}, v_{c 2}\right] ; \rho_{3}(v)=\rho(v), v \in\left[v_{c 2},+\infty\right) \text {. }
$$

Based on the results of calculations in the $(n, \rho)$ plane, graphs $\left(n_{i}(v), \rho_{i}(v)\right), / i=1,2,3 /$ are constructed.

5. For each jamming mode, according to formula (22), the corresponding values of the reduced potential are calculated in parametric form

$$
\begin{gathered}
\Pi_{1}(v)=\widetilde{\Pi}^{*}(v), v \in\left[0, v_{c 1}\right] ; \Pi_{2}(v)=\widetilde{\Pi}^{*}(v), v \in\left[v_{c 1}, v_{c 2}\right] \\
\Pi_{3}(v)=\widetilde{\Pi}^{*}(v), v \in\left[v_{c 2},+\infty\right) .
\end{gathered}
$$

Based on the results of calculations in the $(n, \Pi)$ plane, graphs $\left(n_{i}(v), \Pi_{i}(v)\right), / i=1,2,3$ / are constructed. Based on these graphs, the stability of various modes of vibration machine motion is estimated.

\subsection{Computational experiment}

Calculations are carried out for the following values of dimensional parameters:

$$
\begin{gathered}
m_{p l}=0.04 \mathrm{~kg} ; l=0.1 \mathrm{~m} ; c=10000 \mathrm{~N} / \mathrm{m} ; M=3.96 \mathrm{~kg} \\
\tilde{b}=10 \mathrm{~N} \cdot \mathrm{s} / \mathrm{m} ; \widetilde{\beta}=0.05 \mathrm{~N} \cdot \mathrm{s} / \mathrm{m}
\end{gathered}
$$

They correspond to the following values of dimensionless parameters

$$
b=0.05 ; \beta=0.025 ; \varepsilon=0.01 ; \chi=\varepsilon b / \beta=0.02 ; \widetilde{I}_{O}^{(p n)}=0.01 .
$$

Bifurcation shaft rotational velocities and corresponding jamming velocities

$$
n_{1}=1.440, v_{c 1}=1.242 ; n_{2}=9.012, v_{c 2}=1.001 \text {. }
$$

Figure 3 shows graphs of the angular velocities of the pendulum jamming (Fig. 3, a), the oscillation amplitudes of the platform (Fig. 3, b), and the generalized potential (Fig. 3, c).

Stability or instability of jamming modes is determined by the graph of the reduced potential constructed for various jamming modes (Fig. 3, c). The dotted line shows the second jamming mode, which is always unstable. 
Since no other steady-state modes of system motion are found, then for the system under consideration it is possible to construct a complete bifurcation diagram in which the shaft rotation velocity is selected by the bifurcation parameter.

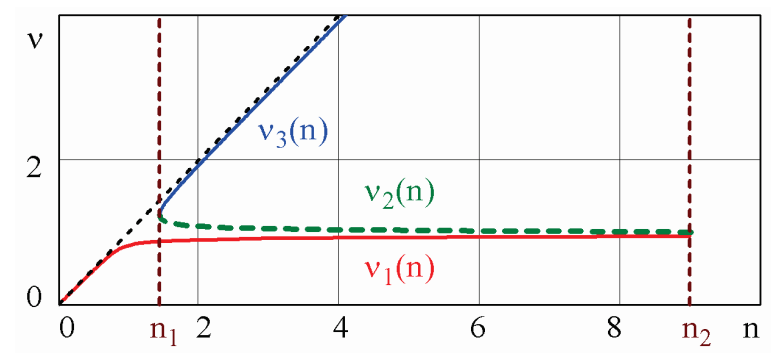

a

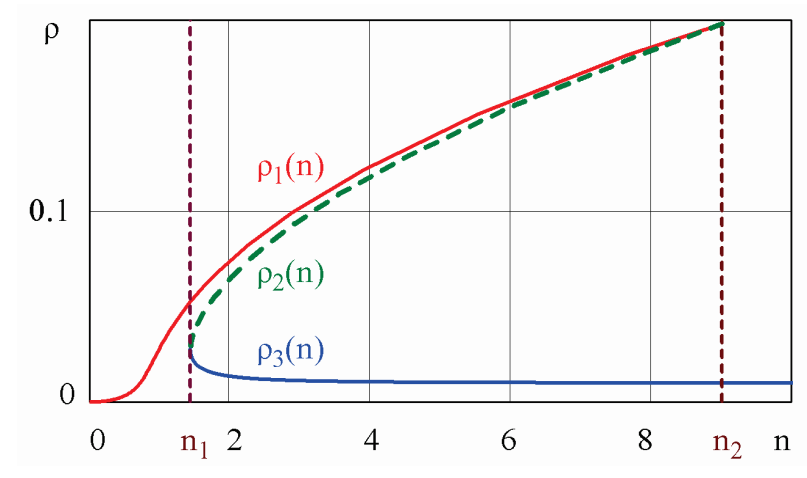

b

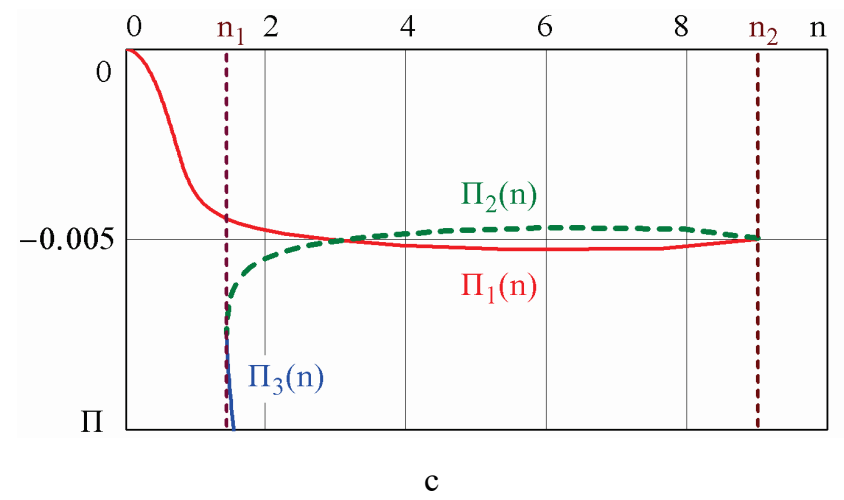

Fig. 3. Bifurcation diagrams of motions constructed by: a - angular velocity of the pendulum jamming; $b$ - amplitude of the oscillations of the platform; $\mathrm{c}$ - generalized (reduced) potential.

Such a diagram can be represented in the form of graphs depicted in Figure 3a, Figure $3 \mathrm{~b}$ or Figure 3, c. But only according to Figure 3, it is possible to determine that in the range of angular velocities of rotation of the shaft:

1) $\left(0, n_{1}\right)$ jamming mode $v_{1}$ is globally asymptotically stable;

2) $\left(n_{1}, n_{2}\right)$ jamming modes $v_{1}, v_{3}$ are locally asymptotically stable, and jamming mode $v_{2}$ is unstable;

3) $\left(n_{2},+\infty\right)$ jamming mode $v_{3}$ is globally asymptotically stable. 


\subsection{Discussion}

The conducted studies show the effectiveness of the developed methodology for studying the modes of jamming of pendulums (balls, rollers) in systems similar to this one. The technique is based on the energy method, the theory of bifurcations of motions, and the idea of a parametric solution to the problem.

Application of the technique to a specific mechanical system shows that the system has only such steady-state modes of motion in which the pendulum rotates around the longitudinal axis of the shaft with a constant angular velocity and lags behind the shaft. In this case, there are two bifurcation velocities of shaft rotation $n_{1}, n_{2}$, and they are of the resonance $\left(1<n_{1}<<n_{2}\right)$. At shaft velocities:

- less than $n_{1}\left(0<n<n_{1}\right)$, there is a unique jamming frequency $v_{1}$, with $0<v_{1}<1$;

- exceeding $n_{1}$, but less then $n_{2} \quad\left(n_{1}<n<n_{2}\right)$, there are three jamming frequencies $v_{1,2,3}$, such that $0<v_{1}<v_{2}<v_{3}<n$ (and always $1<v_{2}<v_{3}$ );

- exceeding $n_{2}\left(n>n_{2}\right)$, there is a single jamming frequency $v_{3}$, such that $1<<v_{3}<n$;

- in the range of angular velocities of shaft rotation $\left(0, n_{1}\right)$, the jamming mode $v_{1}$ is globally asymptotically stable;

- in the range of angular velocities of shaft rotation $\left(n_{1}, n_{2}\right)$, the jamming modes $v_{1}, v_{3}$ are locally asymptotically stable, and what mode is established depends on the initial conditions, and the jamming mode $v_{2}$ is unstable;

- in the range of angular velocities of shaft rotation $\left(n_{2},+\infty\right)$, the jamming mode $v_{3}$ is globally asymptotically stable.

The smallest angular velocity of the pendulum jamming is close to resonance. Therefore, the corresponding jamming mode of the pendulum can be used to excite resonant vibrations in vibrating machines. The highest frequency of a pendulum jamming is close to the shaft rotation velocity. This mode can be used to excite non-resonant vibrations in vibration machines.

It should be noted that the developed technique is applicable only in the case of isotropic supports.

In the future, it is planned to investigate the existing modes of motion of two-mass and three-mass resonant vibration machines with a vibration exciter in the form of a pendulum.

\section{Conclusions}

1. The conducted studies show the effectiveness of the developed methodology for studying the jamming modes of pendulums (balls, rollers) in systems similar to this one. The technique is based on the energy method, the theory of bifurcations of motions, and the idea of a parametric solution to the problem.

2. Using the methodology for a particular system, all steady-state modes of motion are found in which the pendulum rotates at a constant angular velocity. It is established that these are modes of the pendulum jamming.

Depending on the shaft rotation velocity, there may be one or three possible velocities of the pendulum jamming. When there is only one angular velocity, the pendulum is jammed, the corresponding mode of motion is globally asymptotically stable. When there are three jamming velocities, locally asymptotically stable modes with the smallest and highest jamming velocities of the pendulum.

3. The jamming mode of the pendulum with the lowest angular velocity (close to the resonant) can be used to excite resonant vibrations in vibration machines. The highest 
frequency of a pendulum jamming is close to the shaft rotation velocity. This mode can be used to excite non-resonant vibrations in vibration machines.

\section{References}

1. Filimonikhin, G., Yatsun, V. (2015). Method of excitation of dual frequency vibrations by passive autobalancers. Eastern-European Journal Of Enterprise Technologies, 4(7(76)), 9-14. doi: http://dx.doi.org/10.15587/1729-4061.2015.47116

2. Filimonikhin, G., Filimonikhina, I., Ienina, I., Rahulin, S. (2019). A procedure of studying stationary motions of a rotor with attached bodies (auto-balancer) using a flat model as an example. Eastern-European Journal Of Enterprise Technologies, 3(7 (99)), 43-52. doi: http://dx.doi.org/10.15587/1729-4061.2019.169181

3. Artyunin A.I., Eliseev S.V., Sumenkov O.Y. (2019). Experimental Studies on Influence of Natural Frequencies of Oscillations of Mechanical System on Angular Velocity of Pendulum on Rotating Shaft. In: ICIE 2018, 159-166. doi: https://doi.org/10.1007/9783-319-95630-5_17

4. Filimonikhin, G., Yatsun, V., Filimonikhina, I., Ienina, I., Munshtukov, I. (2019). Studying the load jamming modes within the framework of a flat model of the rotor with an autobalancer. Eastern-European Journal Of Enterprise Technologies, 5(7 (101)), 51-61. doi : http://dx.doi.org/10.15587/1729-4061.2019.177418

5. Yatsun, V., Filimonikhin, G., Podoprygora, N., Pirogov, V. (2019). Studying the excitation of resonance oscillations in a rotor on isotropic supports by a pendulum, a ball, a roller. Eastern-European Journal of Enterprise Technologies, 6(7 (102)), 32-43. doi: http://dx.doi.org/10.15587/1729-4061.2019.182995

6. Ryzhik, B., Sperling, L., Duckstein, H. (2004). Non-synchronous Motions Near Critical Velocitys in a Single-plane Autobalancing Device. Technische Mechanik, 24, 25-36.

7. Lu, C.-J., Tien, M.-H. (2012). Pure-rotary periodic motions of a planar two-ball autobalancer system. Mechanical Systems and Signal Processing, 32, 251-268. doi: https://doi.org/10.1016/j.ymssp.2012.06.001

8. Green, K., Champneys, A. R., Lieven, N. J. (2006). Bifurcation analysis of an automatic dynamic balancing mechanism for eccentric rotors. Journal of Sound and Vibration, 291 (3-5), 861-881. doi: https://doi.org/10.1016/j.jsv.2005.06.042

9. Strauch, D. (2009). Classical Mechanics: An Introduction. Springer-Verlag Berlin Heidelberg, 405. doi: https://doi.org/10.1007/978-3-540-73616-5

10. Ruelle, D. (1989). Elements of Differentiable Dynamics and Bifurcation Theory. Academic Press, 196. doi: https://doi.org/10.1016/c2013-0-11426-2 